\title{
A Global Magnetohydrodynamic Simulation of the Origin and Evolution of Magnetic Flux Ropes in the Magnetotail
}

\author{
Raymond J. WALKER ${ }^{1}$ and Tatsuki OGINO ${ }^{2}$ \\ ${ }^{1}$ Institute of Geophysics and Planetary Physics, University of California, Los Angeles CA 90095-1567, U.S.A. \\ ${ }^{2}$ Solar Terrestrial Environment Laboratory. Nagoya University, Toyokawa, Aichi 442, Japan
}

(Received October 2, 1995; Revised February 19, 1996; Accepted March 13, 1996)

\begin{abstract}
We have used a three dimensional global magnetohydrodynamic simulation to model the response of the magnetosphere to an interplanetary magnetic field (IMF) with $B_{z}<0$ and $B_{y} \neq 0$ in order to study the origin and evolution of magnetic flux ropes in the magnetotail. The southward IMF leads to dayside magnetic reconnection followed by reconnection on closed plasma sheet field lines. When there initially was no IMF $B_{y}$ in the plasma sheet, reconnection led to the formation of a plasmoid composed of a quasitwo dimensional closed magnetic loop structure. When IMF $B_{y}$ reached the equatorial region the quasitwo dimensional plasmoid became a magnetic flux rope. For IMF $B_{y} \neq 0$ initially in the plasma sheet the reconnection immediately led to the formation of a flux rope structure. The pitch of the flux rope field lines is determined by the amount of $B_{y}$. For the case with no initial $B_{y}$ in the plasma sheet the pitch was relatively large while when $B_{y} \neq 0$ initially the flux rope resembled a flux tube lying across the tail. Flux ropes in the magnetotail consist of closed, open and IMF field lines. The open and IMF field lines in the flux rope become attached to the IMF when closed field lines in the flux rope reconnect with IMF field lines at the flank magnetopause. Flux ropes can move tailward before all of the closed field lines have reconnected. This is primarily caused by the tension on IMF field lines which drape over the flux rope when lobe field lines reconnect. However the tailward velocity is less for flux ropes than for plasmoids because of the connection to the Earth. In the simulation closed flux rope field lines are found over $100 R_{E}$ down the tail.
\end{abstract}

\section{Introduction}

It has been over a decade since observations confirmed the existence of plasmoids moving tailward during magnetospheric substorms (Hones et al., 1984). Shortly after that Sibeck et al. (1984) in the distant tail and Elphic et al. (1986) in the near Earth tail reported the presence of the three dimensional magnetic structures frequently called magnetic flux ropes. Hughes and Sibeck's (1987) plasmoid formation model predicts that plasmoids form as flux ropes rather than as quasi-two dimensional closed loop structures. Observational studies also have suggested that plasmoids frequently are magnetic flux ropes (Elphic et al., 1986; Sibeck, 1990; Moldwin and Hughes, 1991, 1992; Kivelson et al., 1993; Frank et al., 1994; Kawano et al., 1994). Observationally flux ropes most frequently have been distinguished from plasmoids by the presence of a large magnetic spike in the center of the structure. This is in addition to the bipolar signature in the component of $\boldsymbol{B}$ normal to the axis of the rope. In the magnetotail the spike is usually in the $B_{y}$ component while the bipolar signature is usually in $B_{z}$ but other configurations have been reported (see Moldwin and Hughes $(1991,1992)$ for examples). This signature has been interpreted as indicating that the spacecraft passed near the center of a flux rope oriented approximately parallel to the $y$ direction (e.g. Elphic et al., 1986; Moldwin and Hughes, 1991). $B_{y}$ in the flux rope has the same sign as $B_{y}$ in the interplanetary magnetic field (IMF) (Moldwin and Hughes, 1992; Slavin et al., 1995).

Simulation studies of magnetotail dynamics have found that twisted flux ropes form in the magnetotail (Birn and Hesse, 1990, 1991; Ogino et al., 1990; Hesse and Birn, 1991). Ogino et al. (1990) used a global magnetohydrodynamic (MHD) simulation to model the magnetosphere when the interplanetary 
magnetic field (IMF) has $B_{z}<0$ and $B_{y} \neq 0$ and found the flux ropes had structures similar to those inferred from the observations. In particular the core field had the same sign as IMF $B_{y}$. In addition the simulated flux ropes were found to be filamentary with IMF, open and closed field lines threading the flux tube (Ogino et al., 1990; Birn and Hesse 1990, 1991; Hesse and Birn, 1991).

In this paper we have used a three dimensional global MHD model to further examine the origin and evolution of flux ropes in the magnetotail. We examine the formation of flux ropes and their temporal evolution by analyzing the results from two simulations. First we consider a case in which there was no initial $B_{y}$ in the plasma sheet and in the second example we consider a case in which $B_{y} \neq 0$ in the plasma sheet when IMF was turned southward.

\section{Case 1: No initial plasma sheet $B_{y}$}

For this study we have used a high resolution global MHD code developed by Ogino et al. (1992). Since the details of this model have been published we will describe it only briefly. In the code used in Case 1 we solve the resistive MHD equations and Maxwell's equations as an initial value problem on a rectangular grid with $(212 \times 72 \times 142)$ points and a mesh size of $0.4 R_{E}$. We solve the differential equations by using a modified version of the Leapfrog scheme which is a combination of the Leapfrog scheme and the two step Lax-Wendroff scheme. The simulation parameters are fixed to solar wind values at the upstream edge of the simulation box with free boundary conditions at the sides and back. Since the simulation includes only the dawn half of the magnetosphere, symmetry boundary conditions are used to

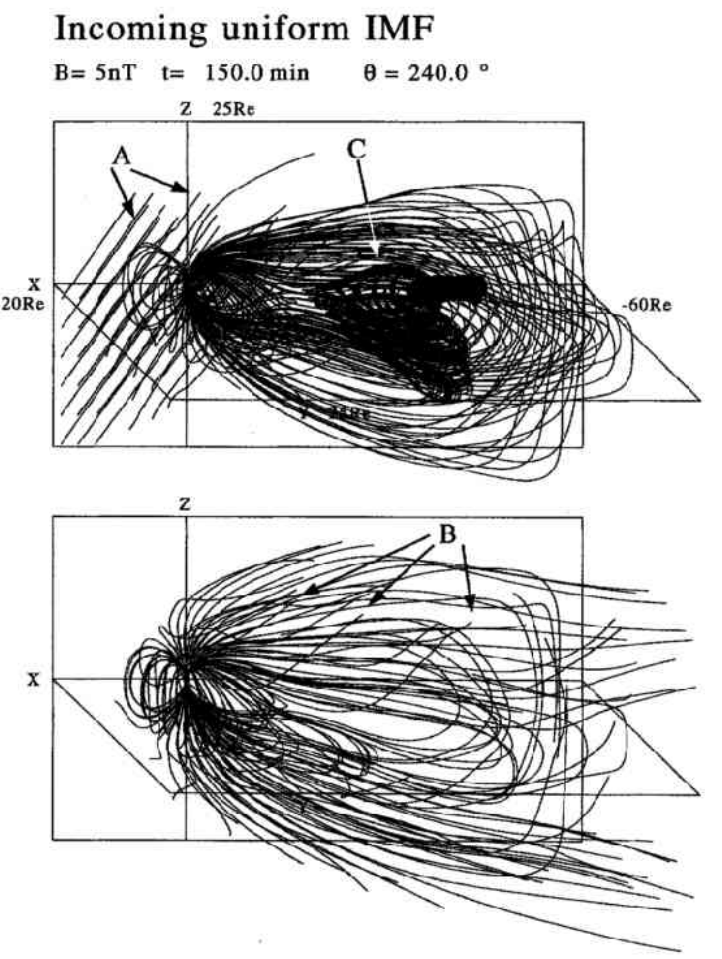

Fig. 1. Magnetic field lines at $T=150 \mathrm{~min}$ or $60 \mathrm{~min}$ after the southward and dawnward IMF entered the simulation box in Case 1. The field lines on the top were calculated starting at the equator while those on the bottom were started at the Earth. (A) indicates IMF field lines, (B) indicates reconnected field lines in the tail lobes and (C) indicates plasmoid field lines. $\theta$ gives the clock angle of the IMF in the $Y Z$ plane with $0^{\circ}$ for duskward, $90^{\circ}$ for northward, $180^{\circ}$ for dawnward and $270^{\circ}$ for southward. 
construct the dusk half of the magnetosphere given the values of the parameters on the dawn half. Fixed ionospheric boundary conditions are applied within $5.5 R_{E}$ of the Earth. For these simulations the solar wind velocity was $300 \mathrm{~km} / \mathrm{s}$, the density was $5 \mathrm{~cm}^{-3}$ and the temperature was $2 \times 10^{5} \mathrm{~K}$.

Initially the simulation was run for $90 \mathrm{~min}$ in real time without an IMF in order to form a "quiet" magnetosphere. However we should note that at the end of this interval the magnetosphere had not reached a steady state and was still gradually evolving. There was no IMF $B_{y}$ in the plasma sheet at the end of the initial run. Next an IMF with southward and dawnward components $\left(B_{z}=-4.3 \mathrm{nT}\right.$ and $\left.B_{y}=-2.5 \mathrm{nT}\right)$ entered the upstream boundary of the simulation box.

Figure 1 shows magnetic field lines at $T=150 \mathrm{~min}$ ( $60 \mathrm{~min}$ after the southward and dawnward IMF entered the simulation). Note that both times will be used throughout this paper with the time in parentheses being the time after the southward turning of the IMF. We calculated field lines in the bottom of the figure starting at the Earth while for those on the top the field lines were calculated starting at the
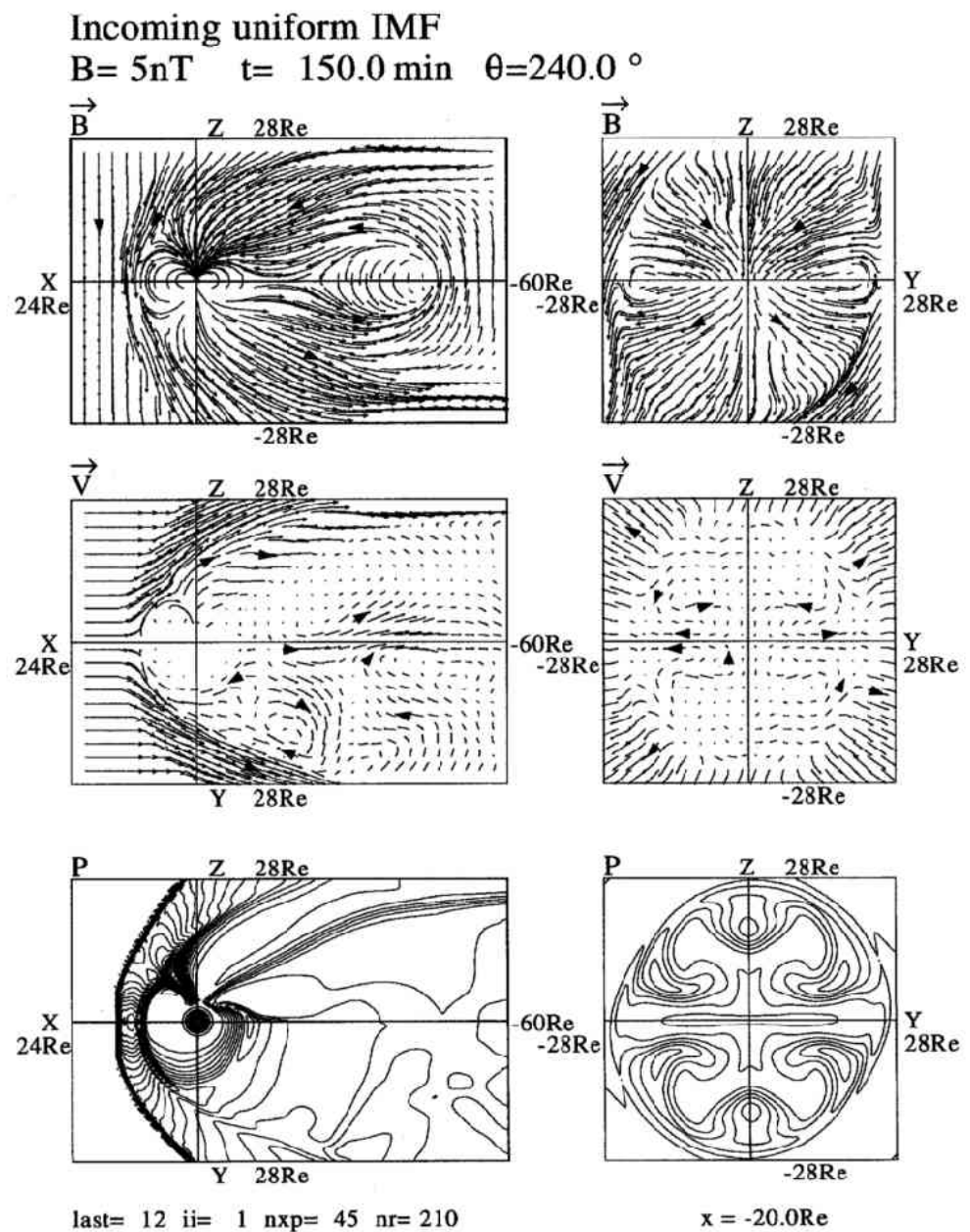

Fig. 2. Magnetic field vectors $(\vec{B})$, velocity vectors $(\vec{V})$ and pressure contours $(P)$ at $T=150 \mathrm{~min}(60 \mathrm{~min})$ in Case 1 . On the left the magnetic vectors are plotted in the noon-midnight meridian plane, while the velocity vectors and the pressure are plotted so that the noon-midnight meridian plane is the top half of the panel and the equator is the bottom half. The values on the left are plotted in the $Y Z$ plane at $x=-20 R_{E}$. 
equator. Up until this time the sequence of events has been similar to those found when the IMF is purely southward (Walker et al., 1993). When the southward and dawnward IMF (A) reached the dayside magnetopause reconnection began although unlike in the purely southward IMF case the reconnection was not in the flow stagnation region (see Fig. 7 of Ogino et al. (1986)). In the bottom panel of Fig. 1

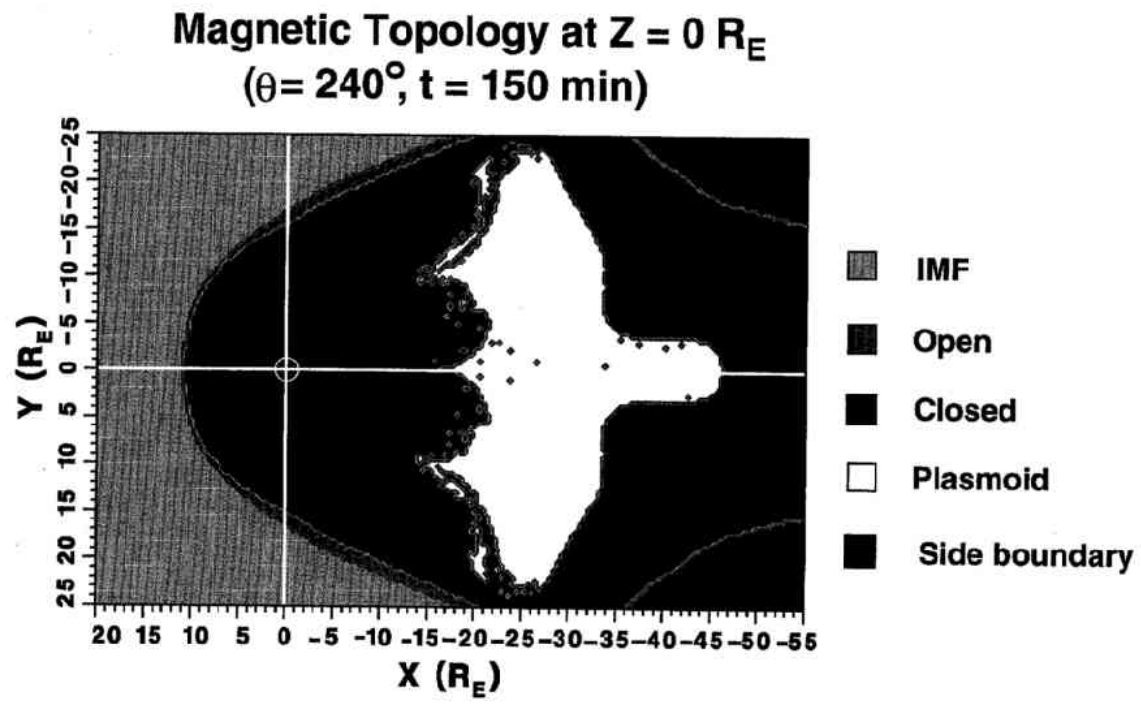

Fig. 3. The configuration of magnetic field lines crossing the equatorial plane at $T=150 \mathrm{~min}(60 \mathrm{~min})$ in Case 1 . Light gray indicates IMF, darker gray open and black closed field lines. White indicates field lines that close on themselves. Field lines in the darkest gray region were too close to the boundary to determine the configuration.

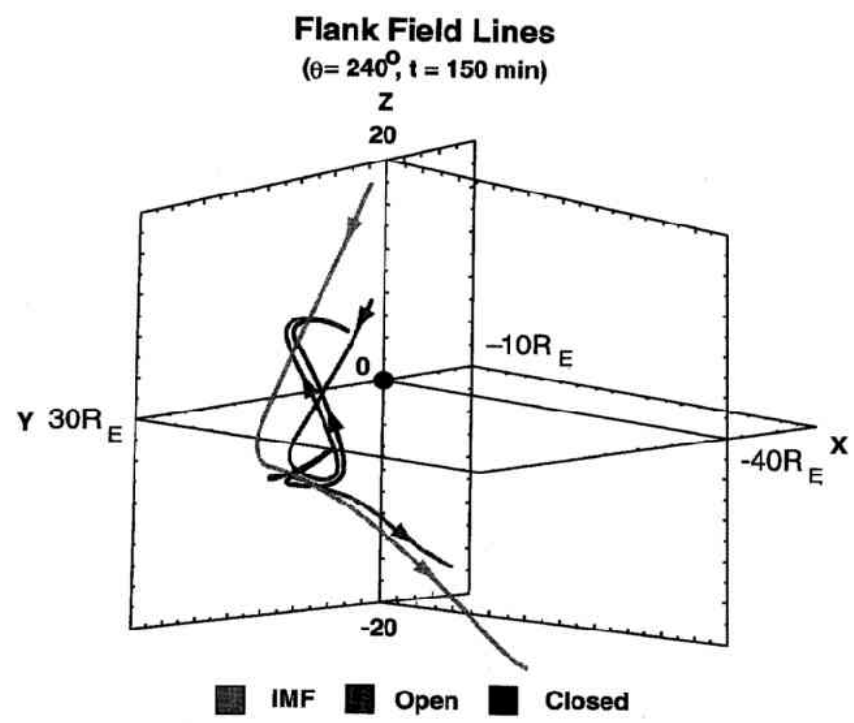

Fig. 4. Magnetic field lines along the dusk flank of the magnetosphere at $T=150 \mathrm{~min}(60 \mathrm{~min})$ in Case 1 . The light gray field line is a magnetosheath field line, the black field line is closed and the dark gray field lines are open. 
reconnected field lines can be seen in the tail lobes (B). Just prior to this time reconnection began in the plasma sheet on closed field lines. A plasmoid (C) can be seen in the top panel of Fig. 1. Note that this is a closed loop structure to a very good approximation.

In Fig. 2 we have plotted magnetic field vectors, velocity vectors and pressure contours. In the left column the magnetic field is plotted in the noon-midnight meridian while the velocity and pressure are plotted so that the top of each panel is the noon-midnight meridian while the bottom is the equatorial plane. The right column gives the values in the $Y Z$ plane at $x=-20 R_{E}$. The magnetic vectors are projections along field lines and the flow vectors are projections along streamlines. In both cases these are projections of three dimensional structures. The plasma sheet reconnection can be seen in both the magnetic field vectors and the plasma flow but the effects of the neutral line can be most easily seen in the flow reversal at about $x=-20 R_{E}$. Note that the plasma mantle flow in the top part of the middle left panel does not reach the equatorial plane. The $Y Z$ panels show that the magnetopause has been twisted clockwise by the IMF $B_{y}$ but in the central part of the tail the configuration remained completely symmetric. For instance the plasma sheet pressure is symmetric with respect to the equator and $B_{y}$ in the plasma sheet is that found in the absence of an IMF $B_{y}$ in which $B_{y}$ reverses across the noon-midnight meridian.

Figure 3 shows another way to display the magnetic configuration. In this figure we have labeled the configuration of magnetic field lines crossing the equatorial plane. IMF field lines are light gray, open field lines with one end in the IMF and the other on the Earth are darker gray and closed field lines are black. Two small regions in the tail in which it was difficult to determine the nature of the field have been shaded with the darkest gray. The plasmoid is white since in this region the field lines close on themselves. At this time the plasmoid structure does not extend all of the way across the tail to the magnetopause. Note that there is a band of dark gray along the magnetopause. This indicates the presence of open field lines along the entire magnetopause. The reason for this can be seen in Fig. 4 where four dusk flank field lines

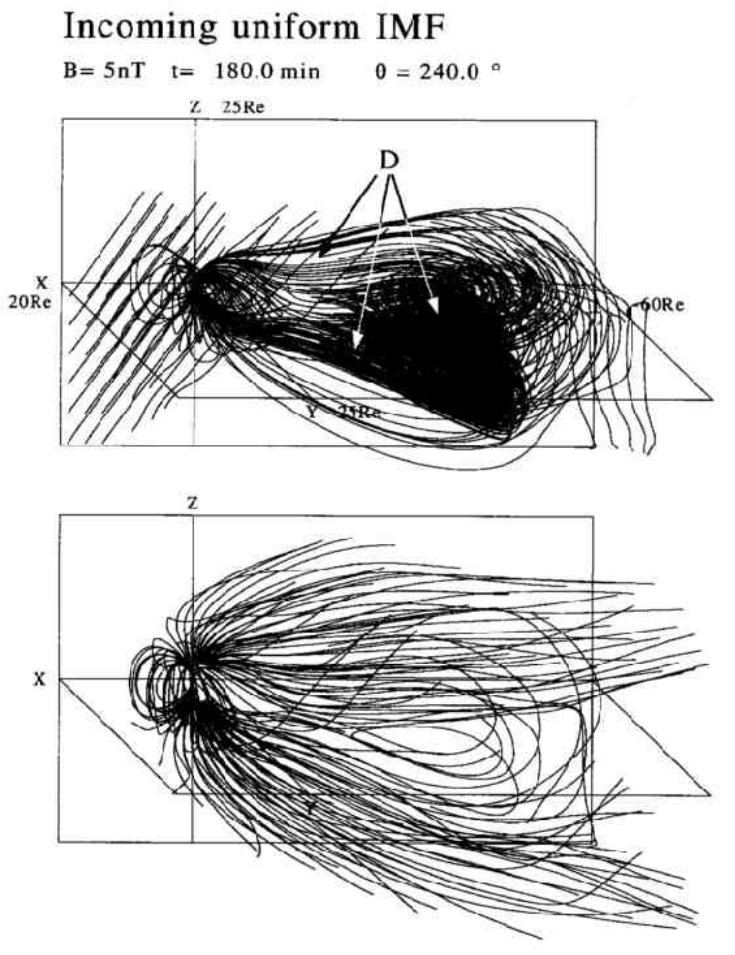

Fig. 5. The same format as Fig. 1 for $T=180 \mathrm{~min}(60 \mathrm{~min})$. $\mathrm{D}$ indicates the magnetic flux rope. 
have been plotted. The light gray line represents an IMF field line in the magnetosheath. This field line which did not participate in magnetopause reconnection was bent as it was convected around the magnetopause obstacle (see Chen et al. (1993) for a discussion of the configuration of the magnetosheath field). The black field line is typical of the closed magnetospheric field on the flanks. The dark gray open field lines form when the magnetosheath field reconnects with the magnetospheric field.

Thirty minutes later $(T=180 \mathrm{~min}(90 \mathrm{~min}))$ the closed loop plasmoid has evolved into a flux rope (Fig. 5). In the top panel field lines threading the flux rope (D) start in the southern dusk ionosphere, twist around the flux rope and close in the northern dawn ionosphere. In the interval between the two snapshots the configuration of the plasma sheet has changed. In the right panel of Fig. 6 there is now a dawnward $B_{y}$ throughout the plasma sheet (top) and the plasma sheet too has twisted so that the dawn part has moved northward and the dusk part has moved southward (bottom). In the center panels the convection from the lobes has reached the plasma sheet in a region about midnight $\left(y \approx \pm 10 R_{E}\right)$.

The changes in the magnetospheric configuration are most dramatic in the magnetic topology plot (Fig. 7). Now the flux rope/plasmoid extends all the way to the magnetopause. Most importantly the flux rope region is now light gray indicating that part at least of the flux rope is on IMF field lines as well as
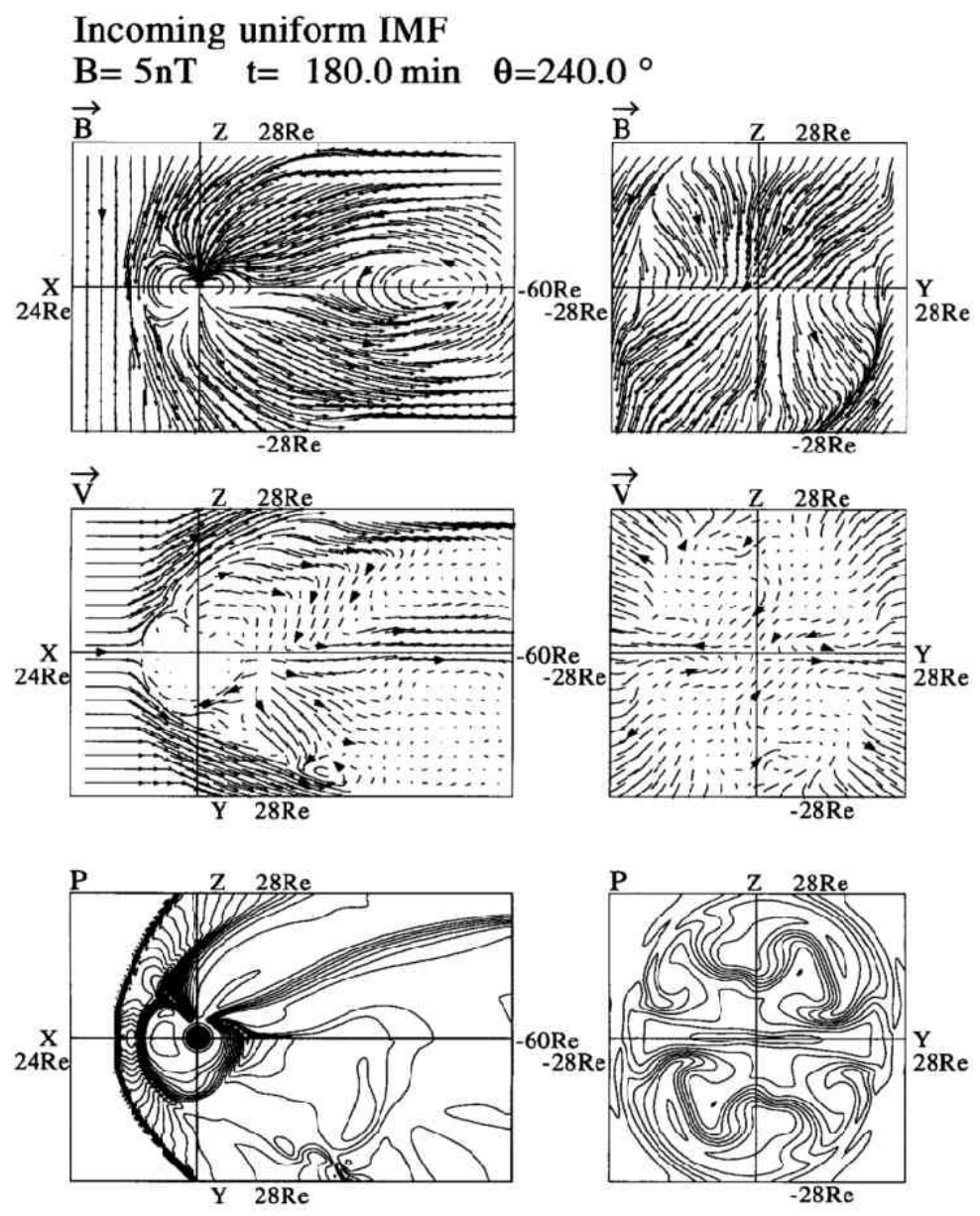

last $=12 \mathrm{ii}=1 \mathrm{nxp}=45 \mathrm{nr}=210$ $x=-20.0 \operatorname{Re}$

Fig. 6. The same format as Fig. 2 for $T=180 \mathrm{~min}(60 \mathrm{~min})$. 


$$
\begin{gathered}
\text { Magnetic Topology at } Z=0 R_{E} \\
\left(\theta=240^{\circ}, t=180 \mathrm{~min}\right)
\end{gathered}
$$

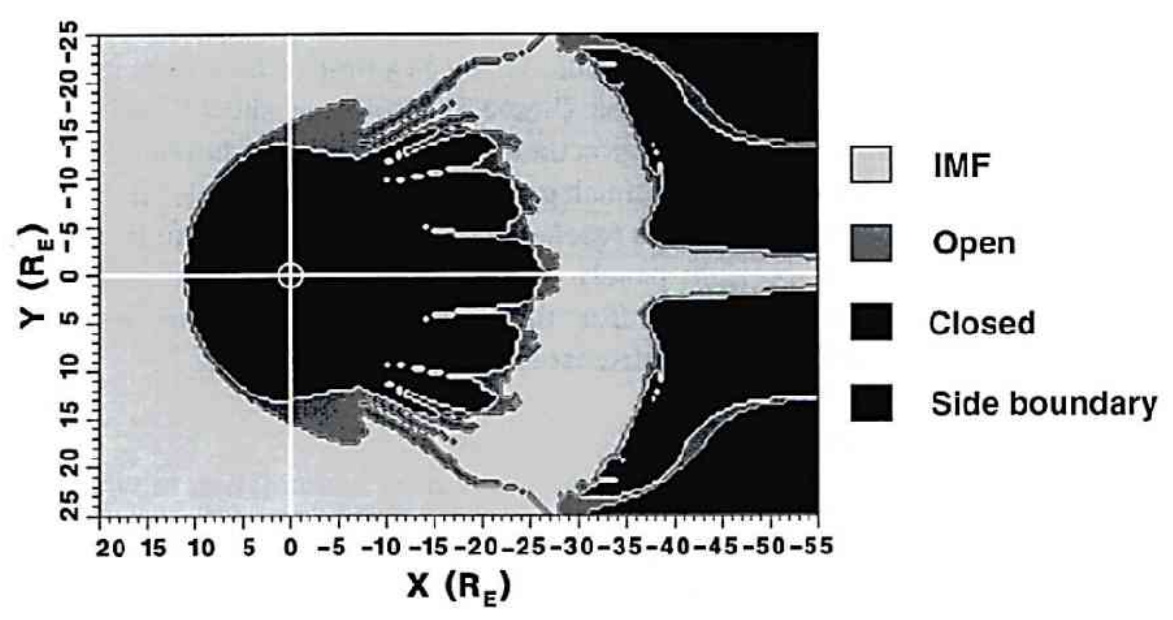

Fig. 7. The same format as Fig. 3 for $T=180 \mathrm{~min}(60 \mathrm{~min})$.
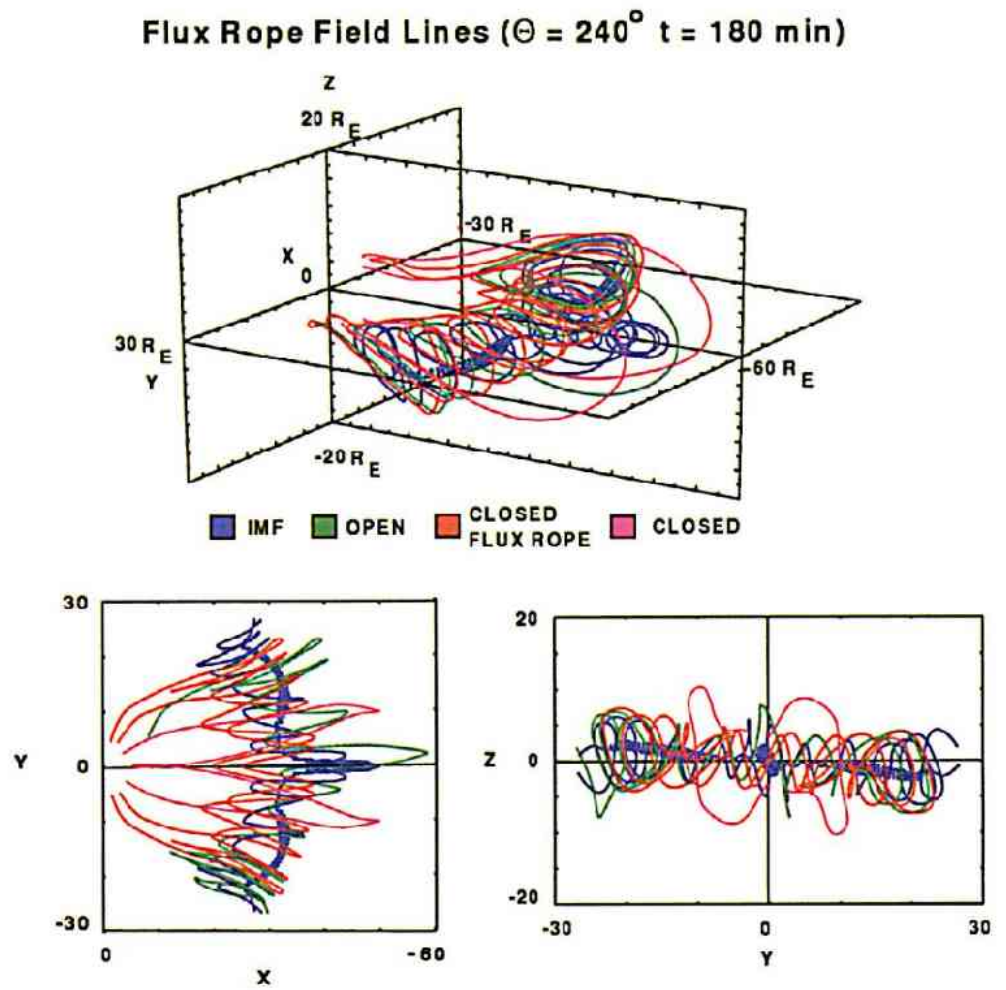

Fig. 8. Magnctic field lines threading the flux rope at $T=180(60 \mathrm{~min})$ in Case 1. Color coding has been introduced to indicate the nature of the field line with red and magenta for closed, green for open and blue for IMF field lines. 
on closed magnetospheric field lines. This can be seen in Fig. 8 in which field lines threading the flux rope have been color coded with IMF field lines blue, open field lines green and closed field lines red. The central part of the flux rope contains field lines that have reconnected at both ends and are attached to the IMF. They are wrapped by green field lines which have undergone reconnection on one side of the magnetosphere only and are still attached to the Earth at the other end. Finally the flux rope has the red field lines which are attached to the Earth at both ends. This was a time of transition in the tail. Most of the closed field in the plasma sheet has reconnected. (Some of the plasma sheet field lines that have not yet reconnected are colored magenta.) Reconnection on tail lobe field lines started a few minutes later. Note that the pitch of the inner most field line is much greater than that of the outer field lines especially near the flanks. This is because while IMF $B_{y}$ has reached the outer plasma sheet only a small amount of $B_{y}$ has reached the equator (Fig. 6 top right panel). Later (not shown) the reconnection at the flank magnetopause completely detaches the flux rope from the magnetosphere and it moves tailward at about $200 \mathrm{~km} / \mathrm{s}$. The tailward motion of flux ropes is discussed in more detail in Case 2.

\section{Case 2: Initially $B_{y} \neq 0$ in the plasma sheet}

As a second example we show results from a interval during a simulation in which the IMF was

\section{Rotation of Incoming IMF \\ $\mathrm{B}=5 \mathrm{nT} \quad \mathrm{t}=675.0 \mathrm{~min} \quad \theta=195.0^{\circ}$}
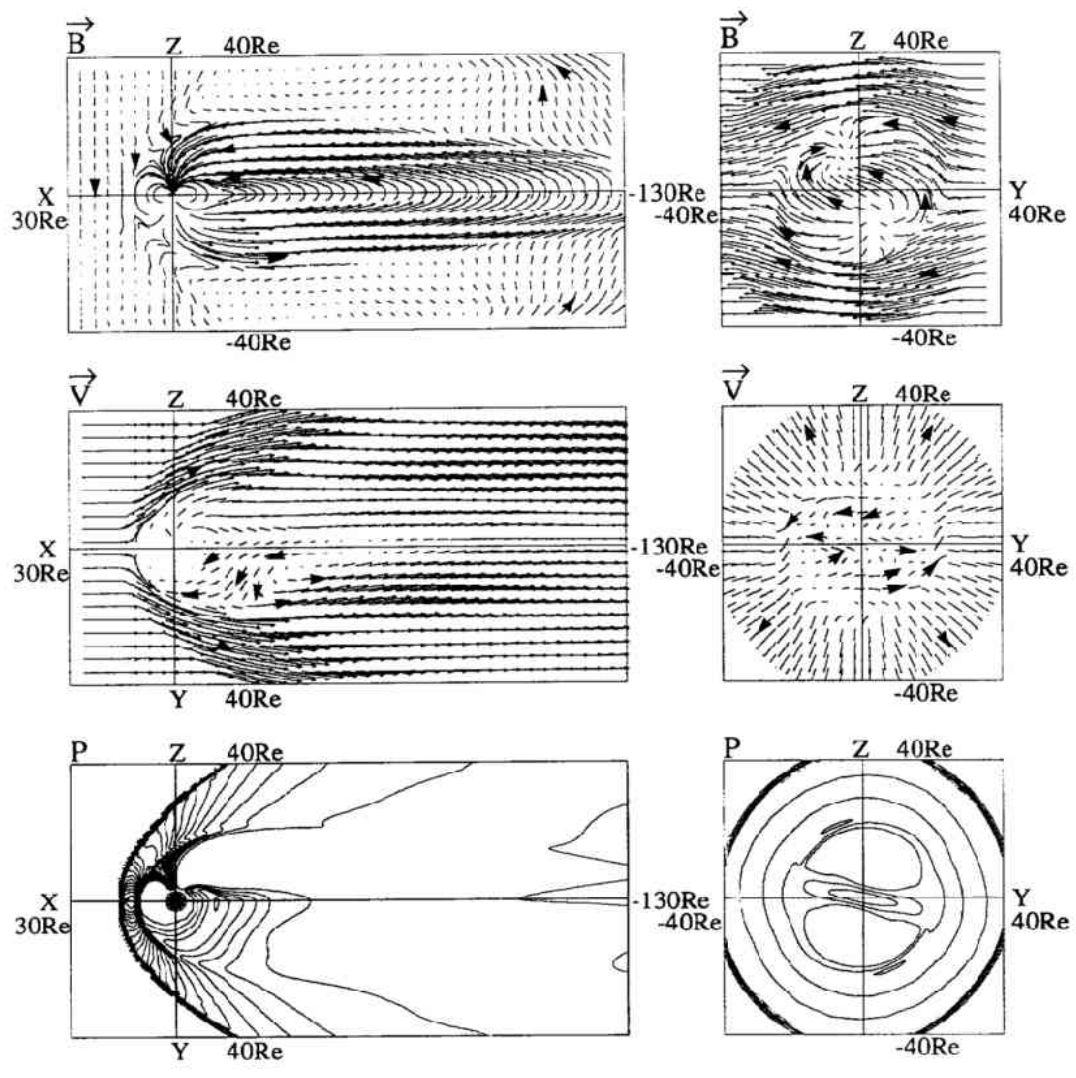

last $=1 \mathrm{ii}=1 \mathrm{nxp}=100 \mathrm{nr}=320$

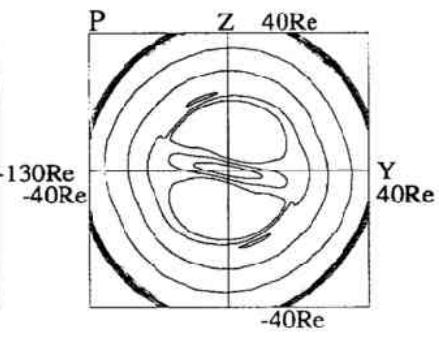

$x=-20.0 \operatorname{Re}$

Fig. 9. Plasma and field parameters at $T=675 \mathrm{~min}(0 \mathrm{~min})$ in Case 2. The format is the same as in Fig. 2 . 
rotated in the $Y Z$ plane in $15^{\circ}$ steps every $15 \mathrm{~min}$. At $T=675 \mathrm{~min}$ the IMF turned southward after having been northward for $3 \mathrm{hr}$ with IMF $B_{y}<0$ for the previous $105 \mathrm{~min}$. For this case we used a $(322 \times 82 \times$ 162 ) point grid with a mesh size of $0.5 R_{E}$ in the simulation. The other solar wind parameters were the same as in Case 1 .

In Fig. 9 we have plotted the plasma parameters at $T=675$ min since the model magnetosphere at this time provides the initial conditions for Case 2. During the $3 \mathrm{hr}$ with northward IMF the magnetospheric configuration was dominated by high latitude reconnection (Ogino et al., 1994). Although we have had high latitude reconnection for three hours the magnetosphere remained open. The high latitude reconnection caused a thickening of the plasma sheet as evidenced by the large $B_{z}$ at the equator in the top left panel and the pressure contours in the lower left panel. The IMF $B_{y}$ caused a twisting of the tail. The plasma sheet (lower right panel) has rotated in a clockwise direction and lies south of the equator at dusk and north of the equator at dawn. Most importantly the plasma sheet has a dawnward $B_{y}$ component (top right panel). There is no reconnection yet in the plasma sheet (top left and left middle panels).

In Fig. 10 we have plotted the plasma parameters 30 min after the southward turning ( $T=705 \mathrm{~min})$. Following the southward turning of the IMF the plasma sheet thinned and the tail field lines became stretched. The thin plasma sheet can be seen in the pressure panels. An examination of the change in the

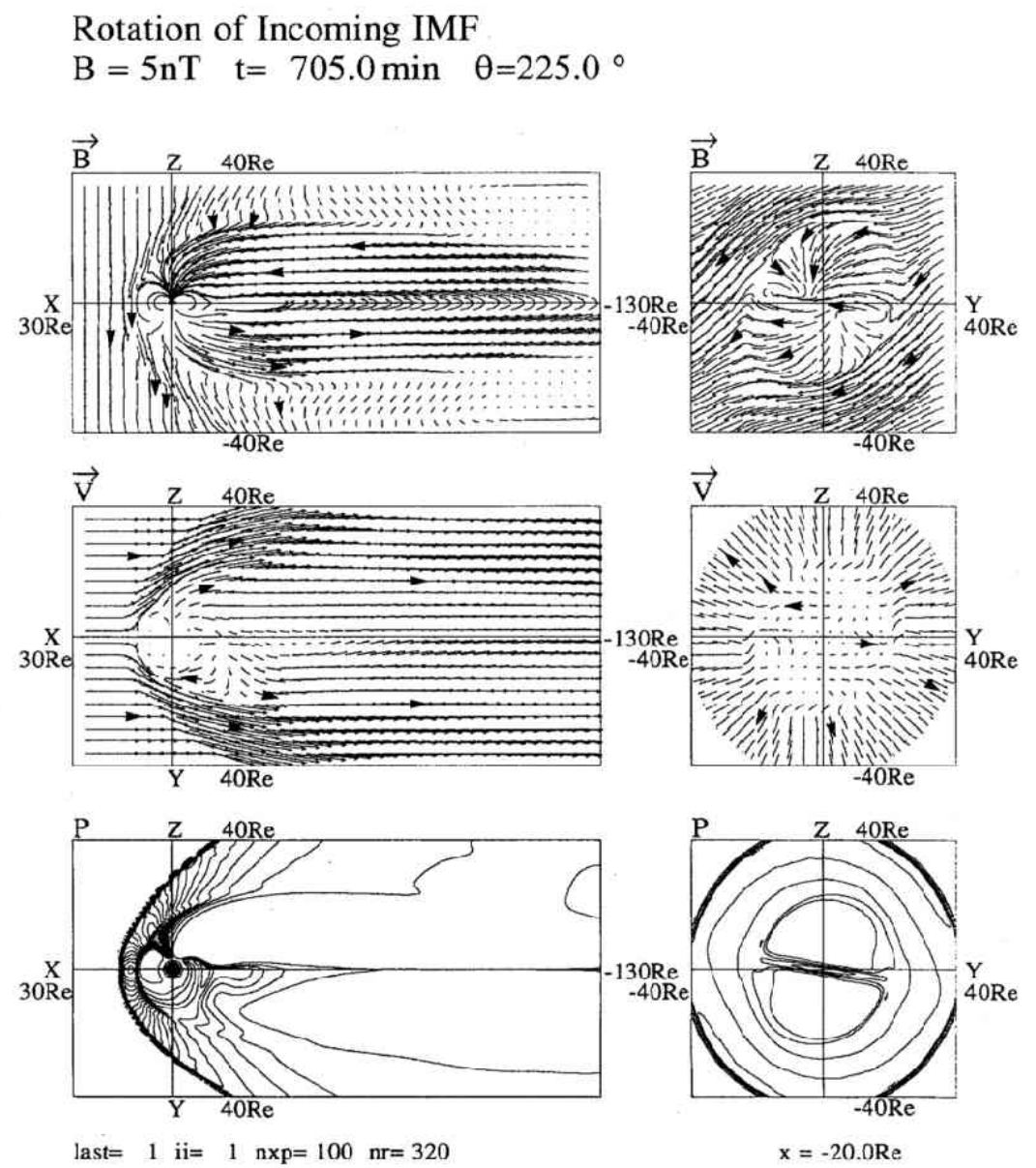

Fig. 10. Plasma and field parameters at $T=705 \mathrm{~min}(30 \mathrm{~min})$ in Case 2 . The format is the same as in Fig. 2 . 
flow pattern between $T=690 \mathrm{~min}(15 \mathrm{~min})$ (not shown) and $T=705 \mathrm{~min}(30 \mathrm{~min}$ ) indicates that reconnection has just started on plasma sheet field lines. In the $Y Z$ magnetic field panel $B_{y}$ has decreased to $B_{y} \approx-10$ $\mathrm{nT}$ in the plasma sheet at $x=-20 R_{E}$.

By $T=720 \mathrm{~min}$ ( $45 \mathrm{~min}$ ) a flux rope has formed. The top of Fig. 11 shows the magnetic configuration in the noon-midnight meridian while on the bottom we have plotted only a few field lines in order to show the structure of the flux rope. In this figure the darker gray shade is used to indicate IMF field lines and black is used to indicate closed field lines. At this time lobe field lines have started to reconnect. The resulting IMF field lines are shown in a lighter shade of gray and drape over the flux rope. Note that even though lobe field lines are reconnecting the flux rope contains field lines with both ends attached to the Earth. This connection remains until all of the closed field lines reconnect at the flank magnetopause. The flux rope has begun to move tailward even though there are closed field lines in the flux rope. This is caused mainly by the tension of draped IMF field lines (Walker et al., 1993).

For this case with $B_{y} \neq 0$ initially the flux rope has very little pitch. It mainly consists of field lines stretched across the magnetosphere. The main twist is on IMF flux rope field lines at the magnetopause.

The flux rope field lines $15 \mathrm{~min}$ later $(T=735 \mathrm{~min}(60 \mathrm{~min}))$ are plotted in Fig. 12. At this time the flux rope has moved approximately $70 R_{E}$ down the tail. There are still closed field lines within the flux

\section{Magnetic Field and Flux Rope Field Lines at $t=720$ and $\Theta=240^{\circ}$}
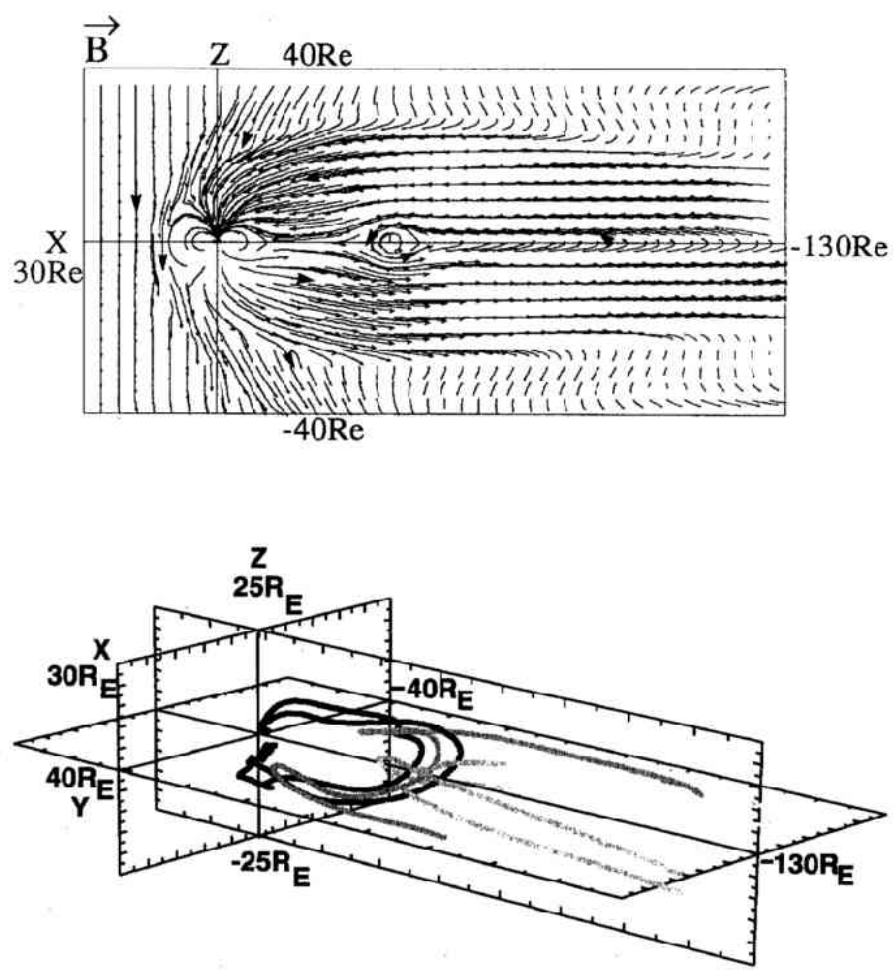

Fig. 11. Magnetic field vectors $(\vec{B})$ in the noon-midnight meridian plane in Case 2 at $T=720 \mathrm{~min}(45 \mathrm{~min})$ (top) and flux rope magnetic field lines. The flux rope magnetic field lines are shaded gray with black representing closed field lines, gray representing IMF field lines threading through the flux rope and light gray representing IMF field lines draped over the flux rope. 

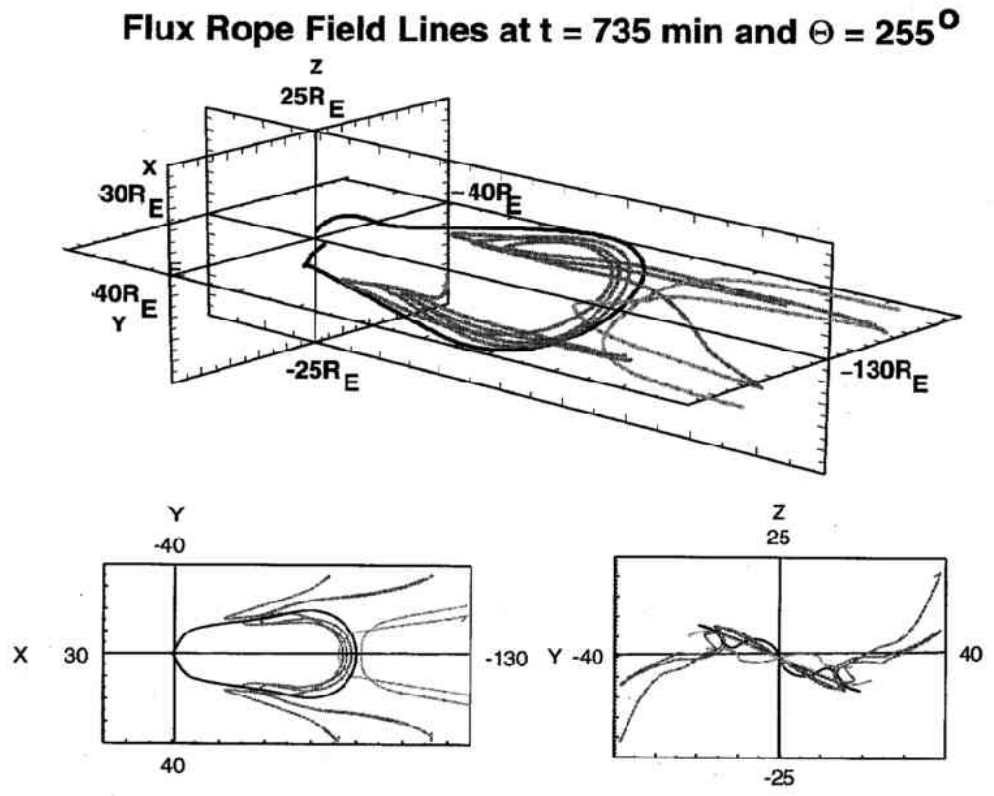

Fig. 12. Flux rope field lines at $T=735(60 \mathrm{~min})$ in Case 2. The format is the same as the bottom of Fig. 11.

Flux Rope Field Lines at $t=750 \mathrm{~min}$ and $\Theta=270^{\circ}$
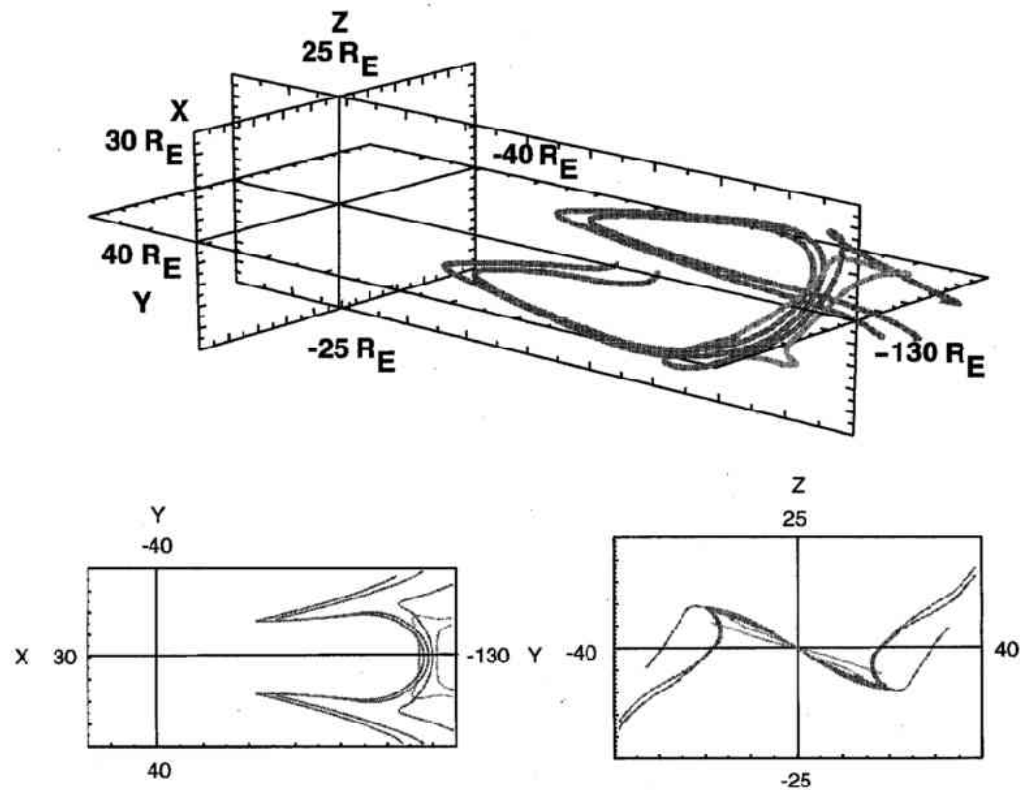

Fig. 13. The same format as Fig. 12 for $T=750 \mathrm{~min}$ ( $75 \mathrm{~min})$. 
rope and the greatest twist is still at the magnetopause. All of the closed field lines didn't reconnect until approximately $T=750 \mathrm{~min}$ ( $75 \mathrm{~min}$ ) (Fig. 13) when the flux rope was over $100 R_{E}$ down the tail. Note that the twist remains near the boundary.

\section{Discussion}

Our global MHD simulations show that when the IMF has $B_{y} \neq 0$ and $B_{z}<0$, magnetic flux ropes form in the magnetotail. This finding is consistent with the results from previous flux rope simulations (Birn and Hesse, 1990, 1991; Ogino et al., 1990, Hesse and Birn, 1990) and observations (Moldwin and Hughes, 1991, 1992; Kivelson et al., 1993; Frank et al., 1994; Slavin et al., 1995). However, in the simulations the flux rope structure did not appear until after IMF $B_{y}$ reached the equatorial region. In Case 1 this occurred after mantle like convection reached the plasma sheet. In Case $2 B_{y}<0$ initially in the plasma sheet and a flux rope like structure formed as soon as tail reconnection began.

The magnetic field and convection patterns in the $Y Z$ plane in Case 1 (Fig. 6 right panels) are consistent with those in the magnetospheric convection model of Khurana et al. (1996) (see their Fig. 5). Following the dayside reconnection, the dawnward IMF convects into the northern dusk and southern dawn lobes. Near the equator the flow turns parallel to the equator and near midnight there is shear in the flow. The magnetic field carried by this flow is responsible for the plasmoid evolving into a flux rope. Prior to the arrival of $B_{y}$ at the plasma sheet, closed field lines which are about to reconnect lie in a plane which is perpendicular to the equator. Since there is no shear on these field lines they reconnect with themselves forming closed loops (Case 1 at $t=150 \mathrm{~min}$ ). When there is an IMF $B_{y}$ in the plasma sheet, the field line becomes skewed and reconnection occurs with another field line displaced in $Y$ (Case 1 at $t=180 \mathrm{~min}$ ). The changes in the magnetic configuration seen in the simulation are essentially those originally proposed by Hughes and Sibeck (1987) and Birn et al. (1989).

Flow Vectors in the YZ Plane at $\mathrm{X}=-120 R_{E}$ and Flux Rope Field Lines

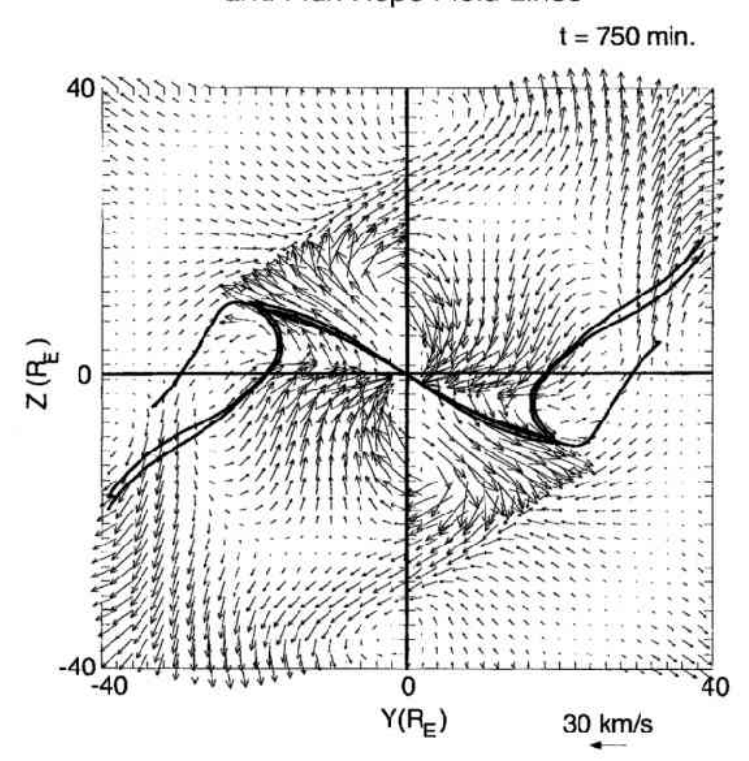

Fig. 14. Flow vectors in the $Y Z$ plane at $x=-120 R_{E}$ for $T=750 \mathrm{~min}$. The flux rope magnetic field lines from Fig. 13 have been projected on the flow vectors. At $T=750 \mathrm{~min}$ the central part of the flux rope passes through $x=-120 R_{E}$. The flow along the flux rope is from the magnetosphere to the magnetosheath. 
The flux ropes in the two cases had somewhat different magnetic configurations. The pitch of the flux rope was much greater in Case 1 than in Case 2 . The central core of Case 1 flux rope was wound very tightly (Fig. 8) while Case 2 flux rope was more like a flux tube lying horizontally across the magnetotail (Figs. 11,12 , and 13). In both cases the flux rope field lines became opened to the solar wind by reconnection along the flank magnetopause. The tail flux ropes therefore should consist of a mixture of IMF, open and closed magnetic field lines. The field topology in Fig. 7 with IMF, open and closed field lines in the flux rope confirms the results in Plate 1 from the magnetotail simulation of Hesse and Birn (1991). The flank magnetopause reconnection occurs preferentially near the equator where the fields are most nearly antiparallel (Fig. 4). In this perfectly symmetrical case this reconnection occurs simultaneously on both the dawn and dusk sides forming the IMF flux rope field lines. However in the real magnetosphere this reconnection probably does not occur on both sides simultaneously resulting in a more complex pattern of IMF field lines.

In Case 2 closed field lines were found in the flux rope until it had moved about $100 R_{E}$ down the tail. This suggests that we should find ionospheric ions (i.e. oxygen) on flux rope field lines even when they are in the distant magnetotail. Frank et al. (1994) have reported bi-directional field aligned streaming of low energy electrons in flux ropes observed on the Geotail spacecraft at $x \approx-130 R_{E}$. This finding is consistent with closed field lines extending to the Earth. Figure 14 shows the flow pattern in the $Y Z$ plane at $x=-120 R_{E}$ at $T=750 \mathrm{~min}$. Superimposed on the flow vectors are the flux rope magnetic field lines from Fig. 13. At this time the central part of the flux rope passed through this plane. There are flows from the magnetosphere to the magnetosheath along the flux rope. Hesse et al. (1995) have suggested that flows of hot plasma sheet plasma along the flux rope into the magnetosheath may contribute to the conversion

Flux Rope Magnetic Signatures

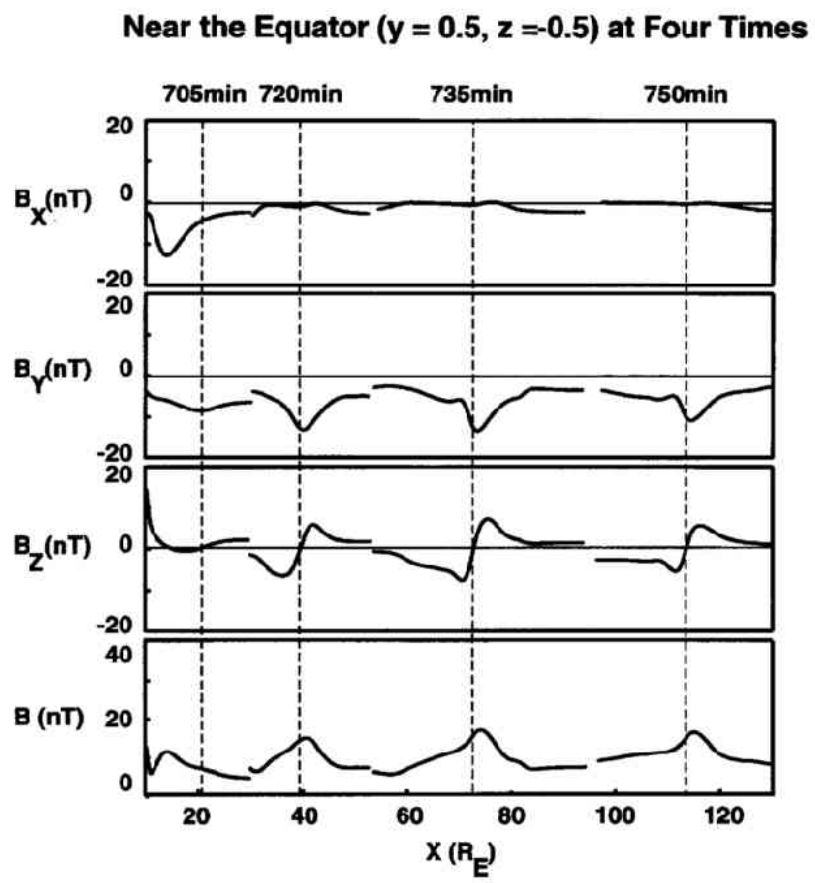

Fig. 15. The magnetic signatures of Case 2 flux ropes. In this figure the magnetic field components and the magnitude at $y=0.5 R_{E}$ and $z=-0.5 R_{E}$ are plotted versus $X$ for four times during Case 2. The dashed lines mark the center of the flux rope at each time and the numbers at the top of the dashed lines give the times. The length of each segment in $X$ was selected to indicate the extent of the flux rope. 
of a near Earth plasmoid into a distant tail flux rope which is depleted of hot plasma in its center.

Figure 15 shows the flux rope magnetic field for the four times studied in Case 2 as a function of $X$. A spacecraft passing near the center of the flux rope will see the classical flux rope signature with a bipolar change in $B_{z}$ and a spike in $B_{y} . B_{y}$ is larger in the flux rope than in the IMF. The magnetic structure of the flux rope didn't change much as it moved tailward. In the distant tail the flux rope has reached a nearly force free configuration. As the plasma exits through the magnetopause the pressure and density in the center of the flux rope decrease (not shown). The magnetic signature of the flux rope has about the same extent in $X\left(\sim 20 R_{E}\right)$ as that inferred from observations by Moldwin and Hughes (1992) $\left(16.3 \pm 13 R_{E}\right)$.

The flux rope starts its tailward motion very slowly. Early in the simulation pressure gradients associated with the formation of the neutral line push the flux rope slowly tailward (Hesse and Birn, 1991; Walker et al., 1993). Even when lobe field lines start to reconnect the movement is slow. This contrasts with the behavior when the IMF is purely southward(Walker et al., 1993). For southward IMF the plasmoid moves rapidly tailward when the lobe field lines reconnect because of the tension on the IMF field lines which drape over it. For $B_{y} \neq 0$ the flux rope is constrained by its connection to the Earth. For instance in the middle tail region $\left(-20 R_{E}<x<-50 R_{E}\right)$ where much of the flux in the flux rope connects to the Earth the tailward velocity is $\sim 120 \mathrm{~km} / \mathrm{s}$. This may be related to the quasi-stagnant plasmoid signatures reported by Nishida et al. (1986) for $x>-100 R_{E}$. The tailward velocity for $x<-70 R_{E}$ is larger $(\sim 280 \mathrm{~km} / \mathrm{s})$. This is less than that inferred from observations by Moldwin and Hughes (1992) $(468 \pm 240 \mathrm{~km} / \mathrm{s})$ but is consistent with the value for tailward bulk flow of $\sim 200 \mathrm{~km} / \mathrm{s}$ measured by Frank et al. (1994). Even in the more distant tail the tailward motion of the flux rope is considerably slower than that of the plasmoids for IMF $B_{y}=0(>400 \mathrm{~km} / \mathrm{s})$ (Walker et al., 1993) because of the connection to the Earth. That closed loop plasmoids move tailward faster than flux ropes was reported by Moldwin and Hughes' (1992) observational study.

The motion in Fig. 15 is different than that reported by Hesse and Birn (1991). In their simulation the velocity of the flux rope increased early in the simulation and then decreased becoming nearly constant late in the simulation (see Fig. 12 of Hesse and Birn (1991)) while the velocity of the flux ropes in Fig. 15 as measured by the spacing between the ropes increased throughout the simulation. The difference may be related to the details of the interaction of the IMF with the flux rope at the magnetopause which are not included in the Hesse and Birn (1991) simulation. The interaction at the flank magnetopause strongly influences the tailward motion of the flux rope. Near the center of the tail pressure gradients and the tension of IMF field lines which drape over the flux rope are sufficient to pull the central part of the flux rope tailward in both the Hesse and Birn (1991) simulation and this simulation. In our simulation the flux rope near the flanks becomes twisted and this kink stays fixed at $X \approx-20 R_{E}$ (Figs. 11 and 12) early in the simulation. The kink occurs at the magnetopause where the IMF flux rope field lines are held in place by the closed flux rope field lines. Thus the flux rope in the magnetosphere assumes a shape similar to a $U$ turned on its side since the central part moves tailward faster than the part near the magnetopause. Later after the last closed field line has reconnected the entire structure is free to move down the tail (Fig. 13).

We would like to thank M. Kivelson for helpful discussions. We also would like to thank R. Richard for helpful discussions and for generating the magnetic topology plots. This paper was completed while one of us (RJW) was a guest at the Max-Planck Institut fuer Extraterrestrische Physik in Berlin. The work at UCLA was supported by Jet Propulsion Laboratory contract JPL 958694, NASA International Solar Terrestrial Physics grant NAG5-1100 and by Air Force Office of Scientific Research contract F49620-95-1-0029. The work at Nagoya University was supported by grants in aid from the Ministry of Education, Science and Culture. Computing support was provided by the San Diego Supercomputer Center and by the Computer Center of Nagoya University.

\section{REFERENCES}

Birn, J. and M. Hesse, The magnetic topology of the plasmoid flux rope in a MHD simulation of magnetotail reconnection, in Physics of Magnetic Flux Ropes, Geophys. Monogr. Ser., 58, edited by C. T. Russell, E. R. Pricst, and L. C. Lee, pp. 655, American Geophysical Union, Washington, D.C., 1990. 
Birn, J. and M. Hesse, MHD simulations of magnetic reconnection in a skewed three-dimensional tail configuration, J. Geophys. Res., 96, A1, 23-24, 1991.

Birn, J., M. Hesse, and K. Schindler, Filamentary structure of a three-dimensional plasmoid, J. Geophys. Res., 94, $241-251,1989$.

Chen, S.-H., M. G. Kivelson, J. T. Gosling, R. J. Walker, and A. J. Lazarus, Anomalous aspects of magnetosheath flow and of the shape and oscillations of the magnetopause during an interval of strongly northward interplanetary magnetic field,J. Geophys. Res., 98, 5727-5742, 1993.

Elphic, R. C., C. A. Cattell, K. Takahashi, S. J. Bame, and C. T. Russell, ISEE 1 and 2 observations of magnetic flux ropes in the magnetotail: FTE's in the plasma sheet, Geophys. Res. Lett., 13, 7,648-7,651, 1986.

Frank, L. A., W. R. Patterson, K. L. Ackerson, S. Kokubun, T. Yamamoto, D. H. Fairfield, and R. P. Lepping, Observations of plasmas associated with the magnetic signature of a plasmoid in the distant magnetotail, Geophys. Res. Lett., 21, 2967-2970, 1994.

Hesse, M. and J. Birn, Plasmoid evolution in an extended magnetotail, J. Geophys. Res., 96, A4, 5683-5696, 1991.

Hesse, M., J. Birn, M. Kuznetsova, and J. Dreher, A model for plasmoid core field formation (abstract), EOS Trans. AGU, 76, 46, F504, 1995.

Hones, E. W., Jr., D. N. Baker, S. J. Bame, W. C. Feldman, J. T. Gosling, D. J. McComas, R. D. Zwickl, J. A. Slavin, E. J. Smith, and B. T. Tsurutani, Structure of the magnetotail at $220 R_{E}$ and its response to geomagnetic activity, Geophys. Res. Lett., 11, 5-7, 1984

Kawano, H., T. Yamamoto, S. Kokubun, K. Tsuruda, A. T. Y. Lui, D. J. Williams, K. Yumoto, H. Hayakawa, M. Nakamura, T. Okada, A. Matsuoka, K. Shiokawa, and A. Nishida, A flux rope followed by recurring encounters with traveling compression regions: Geotail observations, Geophys. Res. Lett., 21, 2891-2894, 1994.

Khurana, K. K., R. J. Walker, and T. Ogino, Magnetospheric convection in the presence of IMF $B_{y}$ : A conceptual model and simulations, J. Geophys. Res., 101, A3, 4907-4916, 1996.

Kivelson, M. G., C. F. Kennel, R. L. McPherron, C. T. Russell, D. J. Southwood, R. J. Walker, K. K. Khurana, P. J. Coleman, C. M. Hammond, V. Angelopoulos, A. J. Lazarus, R. P. Lepping, and T. J. Hughes, The Galileo Earth encounter: Magnetometer and allied measurements, J. Geophys. Res., 98, A7, 11,299-11,318, 1993.

Moldwin, M. B. and W. J. Hughes, Plasmoids as flux ropes, J. Geophys. Res., 96, 14051-14064, 1991.

Moldwin, M. B. and W. J. Hughes, On the formation and evolution of plasmoids: A survey of ISEE 3 Geotail data, J. Geophys. Res., 97, A12, 19,259-19,282, 1992.

Nishida, A., M. Scholer, T. Terasawa, S. J. Bame, G. Glocckler, E. J. Smith, and R. D. Zwickl, Quasi-stagnant plasmoid in the middle tail: A new preexpansion phase phenomenon, J. Geophys. Res., 91, 4245-4255, 1986.

Ogino, T., R. J. Walker, M. Ashour-Abdalla, and J. M. Dawson, An MHD simulation of the effects of the interplanetary magnetic field $B_{y}$ component on the interaction of the solar wind with Earth's magnetosphere during southward IMF, J. Geophys. Res., 91, A9, 10029-10045, 1986.

Ogino, T., R. J. Walker, and M. Ashour-Abdalla, Magnetic flux ropes in 3-dimensional MHD simulations, in Physics of Magnetic Flux Ropes, Geophys. Monogr. Ser., 58, edited by C. T. Russell, E. R. Priest, and L. C. Lee, pp. 669, American Geophysical Union, Washington, D.C., 1990.

Ogino, T., R. J. Walker, and M. Ashour-Abdalla, A global magnetohydrodynamic simulation of the magnetosheath and magnetosphere when the interplanetary magnetic field is northward, IEEE Trans. Plasma Sci., 20, 6,817-6,828, 1992.

Ogino, T., R. J. Walker, and M. Ashour-Abdalla, A global magnetohydrodynamic simulation of the response of the magnetosphere to a northward turning of the interplanetary magnetic field, J. Geophys. Res., 99, A6, 11,027-11,042, 1994.

Sibeck, D. G., Evidence of flux ropes in the Earth's magnetotail, in Physics of Magnetic Flux Ropes, Geophys. Monogr. Ser., 58, edited by C. T. Russell, E. R. Priest, and L. C. Lee, pp. 637, American Geophysical Union, Washington, D.C., 1990.

Sibeck, D. G., G. L. Siscoe, J. A. Slavin, E. J. Smith, S. J. Bame, and F. L. Scarf, Magnetotail flux ropes, Geophys. Res. Lett., 11, 1090-1093, 1984.

Slavin, J. A., C. J. Owen, M. M. Kuznetsova, and M. Hesse, ISEE 3 observations of plasmoids with flux rope magnetic topologies, Geophys. Res. Lett., 1995 (to be submitted).

Walker, R. J., T. Ogino, J. Raeder, and M. Ashour-Abdalla, A global magnetohydrodynamic simulation of the magnetosphere when the interplanetary magnetic field is southward: The onset of magnetotail reconnection, J. Geophys. Res., 98, 17,235$17,249,1993$. 